\title{
Validated UPLC/Q-TOF-MS Method for Determination of Poliumoside in Rat Plasma and Its Application to Pharmacokinetic Study
}

\author{
Hao Qian, Danyi Lu, Wan Li, Xiaotong Zhou, Baojian Wu, Zhiguo Ma* \\ Division of Pharmaceutics, College of Pharmacy, Jinan University, Guangzhou, China \\ Email: bj.wu@hotmail.com, "mzg79@hotmail.com
}

Received 27 January 2016; accepted 12 March 2016; published 15 March 2016

Copyright (C 2016 by authors and Scientific Research Publishing Inc.

This work is licensed under the Creative Commons Attribution International License (CC BY). http://creativecommons.org/licenses/by/4.0/

\section{(c) (i) Open Access}

\begin{abstract}
Poliumoside is the main active constituent of Callicarpa kwangtungensis Chun (CK), a traditional Chinese medicine for management of hemostasis. In this study, a rapid and selective ultra-performance liquid chromatography coupled with quadrupole time-of-flight mass spectrometry (UPLC/ Q-TOF-MS) method was developed and validated to quantify poliumoside in rat plasma. The targeted analytes in rat plasma were prepared through protein-precipitation method using $10 \%$ trichloroacetic acid (TCA). The chromatographic separation was performed on a Waters $\mathrm{BEH} \mathrm{C}_{18}$ column $(2.1 \times 100 \mathrm{~mm}, 1.7 \mu \mathrm{m})$ by acetonitrile-water containing $0.1 \%$ formic acid. The calibration curve was linear over the range of $50-10,000 \mathrm{ng} / \mathrm{mL}\left(\mathrm{r}^{2}>0.99\right)$. The intra-day or inter-day precision was less than $7.97 \%$ and accuracy was within $-7.00 \%-3.36 \%$. The developed method was successfully applied to pharmacokinetic study of poliumoside in rat plasma. Although being rapidly absorbed $\left(T_{\max } \leq 30 \mathrm{~min}\right)$, poliumoside was poorly bioavailable after oral administration (the absolute bioavailability was only $0.69 \%$ ).
\end{abstract}

\section{Keywords}

Poliumoside, UPLC/Q-TOF-MS, Rat Plasma, Pharmacokinetics

\section{Introduction}

Callicarpa kwangtungensis Chun (Verbenaceae, CK), a traditional Chinese medicine, possesses various biolog"Corresponding author.

How to cite this paper: Qian, H., Lu, D.Y., Li, W., Zhou, X.T., Wu, B.J. and Ma, Z.G. (2016) Validated UPLC/Q-TOF-MS Method for Determination of Poliumoside in Rat Plasma and Its Application to Pharmacokinetic Study. American Journal of Analytical Chemistry, 7, 266-274. http://dx.doi.org/10.4236/ajac.2016.73024 
ical activities such as hemostasis and antioxidation [1]. The herbal preparations of "Kang Gong Yan Tablets" and "Kang Gong Yan Capsules" were made up of extracts from CK (the major constituent). As Chinese patent drugs, they have been used in clinic for nearly 20 years to treat gynecological diseases [2].

Poliumoside is the major phenylethanoid glycosides (PhGs) abundantly found in CK [3] and many other plants, such as Cistanche sinensis G. Beck [4], Buddleja officinalis Maxim [5] and Brandisia hancei Hook. F [6]. It is also the chemical maker for both CK and the mentioned herbal preparations on the basis of Pharmacopoeia of the People's Republic of China 2015. Poliumoside also exhibits various activities including antioxidation [7], hemostasis [8], neuroprotection [9] and cell aggregation inhibition [10].

As the potential pharmacological effects, it is necessary to explore the individual pharmacokinetic characteristics of poliumoside for clinical application. Sun et al. developed an ultra-performance liquid chromatography tandem mass spectrometry (UPLC-MS/MS) method for simultaneous determination of crude extract from CK in rat plasma after oral administration [11]. Nevertheless, the multicomponent in herb may impact their individual pharmacokinetic characteristics [12]. The pharmacokinetic behavior of poliumoside after administration of its single form may be different compared with the crude extract from CK. On the other hand, the intravenous administration in rats should also be taken into consideration to confirm the absolute bioavailability of poliumoside. Accordingly, a systematic research on the pharmacokinetics of poliumoside needs to be conducted imminently.

The aim of this article was to develop a rapid and selective UPLC/Q-TOF-MS method for the determination of poliumoside in rat plasma after oral and intravenous administration. This method combines the capabilities of scanning (quadrupole) and high resolution (time of flight). And so far as we know, it has never been used for the analysis of poliumoside in plasma samples. Furthermore, the detailed pharmacokinetic parameters and bioavailability were present for the further research of poliumoside.

\section{Experimental}

\subsection{Chemical and Regents}

Poliumoside (Figure 1, purity > 98\%) and caffeic acid (used as internal standard (IS) were purchased from Chengdu Herbpurity Co. Ltd. (Chengdu, China). Analytical grade trichloroacetic acid (TCA) was purchased from Tianjin Chemical Corporation (Tianjin China). Acetonitrile, methanol and formic acid were all of HPLC grade.<smiles>CC1OC(OCC2OC(OCCc3ccc(O)c(O)c3)C(O)C(OC3OC(C)C(O)C(O)C3O)C2OC(=O)/C=C/c2ccc(O)c(O)c2)C(O)C(O)C1O</smiles>

Caffeic acid<smiles>O=C(O)/C=C/c1ccc(O)c(O)c1</smiles>

Figure 1. The chemical structure of poliumoside and caffeic acid. 


\subsection{Instruments and Conditions}

Chromatographic separation of the analyte was performed on Acquity UPLC system with BEHC 18 column (2.1 $\times 100 \mathrm{~mm}, 1.7 \mu \mathrm{m}$, Waters, Milford, MA, USA). An aliquot of the sample $(5 \mu \mathrm{L})$ was injected into the UPLC system for analysis. Gradient elution was performed with formic acid $(0.1 \%)$ in water (mobile phase A) and acetonitrile (mobile phase B) at a flow rate of $0.4 \mathrm{~mL} / \mathrm{min}$. The gradient elution process was used as follows: $5 \%$ $\mathrm{B}$ at 0 to $1 \mathrm{~min}, 5$ to $95 \% \mathrm{~B}$ at 1 to $3 \mathrm{~min}$, then back to the initial condition within $1 \mathrm{~min}$. Before the next injection, all the samples would undergo a needle wash program to minimize the pollution, the solvent for needle wash containing methanol and water ( $\mathrm{v} / \mathrm{v}, 50: 50)$. The column temperature was maintained at $45^{\circ} \mathrm{C}$.

Mass spectrometry was performed on the Xevo G2 Q-TOF MS (Waters) equipped with the electronic spray ion (ESI) [13] under negative ion mode. The extraction cone, sampling cone and capillary voltages were 4, 40 and $3000 \mathrm{~V}$, respectively. Nitrogen used as cone and desolvation gas were set to 30 and $800 \mathrm{~L} / \mathrm{h}$, respectively. The temperature of source and desolvation were $100^{\circ} \mathrm{C}$ and $250^{\circ} \mathrm{C}$, respectively. Mass spectra were recorded in the range of $m / z 50-1000$. An external reference $2 \mu \mathrm{g} / \mathrm{mL}$ leucine enkephalin $(\mathrm{m} / \mathrm{z} 556.2771)$ was infused at 5 $\mu \mathrm{L} / \mathrm{min}$ to guarantee mass accuracy of the entire assay. The ratio mass-to-charge of poliumoside and IS were 769.2537 and 179.0328 respectively. MS quantitation for pharmacokinetics was performed on the full scan analysis and extracted ion chromatograms using MassLynx version 4.1.

\subsection{Calibration Standards and Quality Control Samples}

Stock solutions of poliumoside $(1 \mathrm{mg} / \mathrm{mL})$ were prepared in saline. Working solutions for control and calibration samples were generated by gradient dilution of the stock solution with methanol:water (60:40). The 10\% TCA used as precipitating agent was prepared in purified water. The IS (caffeic acid) was prepared by dilution of the stock solution $(100 \mu \mathrm{g} / \mathrm{mL})$ with the same solvent to a concentration of $10 \mu \mathrm{g} / \mathrm{mL}$. All of the described solutions were stored at $4^{\circ} \mathrm{C}$ before use.

The calibration solutions were obtained by spiking appropriate volume of working solutions into the aliquots of $100 \mu \mathrm{L}$ blank rat plasma, and the plots were set up at the range of $50-10,000 \mathrm{ng} / \mathrm{mL}(50,100,625,1250$, $2500,5000,10,000 \mathrm{ng} / \mathrm{mL}$ ). As the same to calibration standard, quality control (QC) samples were prepared in three different plasma concentration level $(200,1000,8000 \mathrm{ng} / \mathrm{mL})$. All of the samples were stored at $-20^{\circ} \mathrm{C}$.

\subsection{Sample Preparation}

The rat plasma samples, calibration standard solution and the QC samples were thawed at room temperature before use and put them on the ice for the consideration of stability. Plasma samples were extracted through protein precipitation method by $10 \%$ TCA. In brief, an aliquot of $20 \mu \mathrm{L}$ IS working solution $(10 \mu \mathrm{g} / \mathrm{mL})$ was added into $100 \mu \mathrm{L}$ plasma samples in a $1.5 \mathrm{~mL}$ polypropylenes tube, followed by vortexing for 30 seconds. Subsequently, $80 \mu \mathrm{L}$ of $10 \%$ TCA was added into each tube, vortexing for another 3 min. The mixture was centrifuged under 13,000 g for $15 \mathrm{~min}$ at $4^{\circ} \mathrm{C}$. The supernatant was collected and transferred into a new tube. Then, $5 \mu \mathrm{L}$ of the supernatant was injected into the UPLC/Q-TOF-MS system for the further analysis.

\subsection{Method Validation}

\subsubsection{Selectivity}

The selectivity was verified through the comparison of the chromatograms of blank plasma, blank plasma spiked with poliumoside and caffeic acid, and rat plasma testing samples. To investigate the specificity and make the result dependable, three different batches were tested following the same procedure.

\subsubsection{Linearity and Lower Limit of Quantification (LLOQ)}

To construct the calibration curve, the spiked calibration samples in gradient were analyzed in three consecutive days. Peak area ratios of poliumoside to IS were plotted against analyte concentrations and the date was well fitted by least-square regression using $1 / \mathrm{x}^{2}$ as the weighting factor in the concentration range of $50-10,000$ $\mathrm{ng} / \mathrm{mL}$.

\subsubsection{Accuracy and Precision}

The inter-day accuracy and precision were assessed by analyzing six replicated QC samples at three concentra- 
tion levels (low, middle and high level) on three separated days. The intra-day accuracy and precision were assessed by analyzing the samples on the same day. Concentrations were calculated with calibration curves obtained daily. The relative error (RE) and relative standard deviation (RSD) were used to evaluate the accuracy and precision, respectively.

\subsubsection{The Recovery and Matrix Effect}

The recovery of poliumoside was measured by comparing the peak area of QC samples with those of reference QC solutions reconstituted in blank plasma extracts at low, middle and high level $(n=6)$. The matrix effect was evaluated by calculating the peak area ratio of the response in the presence of matrix ions to the analyte peak response in the absence of matrix ions. Six different lots of blank rat plasma spiked with analyte at three levels were used for the evaluation. The recovery and matrix effect of the IS was measured in the same way.

\subsubsection{Stability}

The stability of poliumoside in rat plasma was investigated by analyzing triplicate QC samples at three concentrations level (low, middle and high), exposed to different conditions. The stability of the spiked samples were evaluated as the following aspects, 1) after three completed freeze-thaw cycles, 2) the exposure of the spiked samples at ice-water bath for $12 \mathrm{~h}, 3$ ) room temperature for $12 \mathrm{~h}$. For stability of poliumoside in processed samples, the prepared QC samples were placed in an auto-sampler at $4^{\circ} \mathrm{C}$ for 3 days. This experiment can clearly determine the stability of the collected samples and the accurate analysis for determination.

\subsection{Pharmacokinetic Study}

Male Sprague-Dawley (SD) rats weighing 220 - 250 g were obtained from Laboratory Animal center of Jinan University (Guangdong, China). Rats were housed in a humidity-controlled room maintained on $12 \mathrm{~h}$ light-dark cycles for 5 days before the experiment. Standard animal food and water were provided ad libitum. Twelve rats were anaesthetized using $10 \%$ chloral hydrate and the jugular vein were cannulated for blood collection before $24 \mathrm{~h}$ of the experiment. Diet was prohibited for $12 \mathrm{~h}$ before the experiment, while water was freely taken. All procedures were approved by the Animal Care and Ethics Committee of Jinan University (Guangzhou, China).

Rats were randomly assigned to two groups (six rats per group). Poliumoside was dissolved in saline and administrated to rats by oral and intravenous injection. Approximately $0.3 \mathrm{~mL}$ blood samples were collected from jugular vein into $1.5 \mathrm{~mL}$ heparinized centrifuge tubes. Time points were set at 2, 5, 10, 20,30, 45, 60, 90, 120, 180, 240, $360 \mathrm{~min}$ for the intravenous group $(10 \mathrm{mg} / \mathrm{kg})$ and 5, 10, 20, 30, 45, 60, 90, 120, 180, 240, 360, 480, $720 \mathrm{~min}$ for the oral group ( $200 \mathrm{mg} / \mathrm{kg}$ ). Blood samples were immediately centrifuged (5000 g, $\left.4^{\circ} \mathrm{C}, 8 \mathrm{~min}\right)$, and $100 \mu \mathrm{L}$ aliquot of supernatant (plasma) was collected and frozen at $-80^{\circ} \mathrm{C}$ before analysis.

\section{Results and Discussion}

\subsection{Method Development and Optimization}

MS parameters were optimized by directly infusing a standard solution into the ESI source. Both positive and negative ion mode were employed to confirm the accurate ions of poliumoside and IS. All analytes showed a better response under the negative mode. Ultimately, the $\mathrm{m} / \mathrm{z} 769.2537$ for poliumoside and 179.0328 for IS were used for the further analysis under negative ion mode. Other parameters have been showed in section 2.2.

To separate the interfering compounds from the poliumoside and IS, different columns and mobile phase compositions were compared for chromatographic separation. The Waters BEH $\mathrm{C}_{18}$ column showed better peak shape and appropriate retention time for the analyte than Phenomenex Kinetex $C_{18}$ column $(2.1 \times 50 \mathrm{~mm}, 2.6$ $\mu \mathrm{m})$. Appropriate ionization and chromatographic behavior were based on the proper mobile phase [14]-[16]. The gradient mobile phase with water (containing $0.1 \%$ formic acid) and acetonitrile greatly shortened the retention time of the analyte. The gradient was verified to be stable and repeatable according to the following test of method validations. The addition of $0.1 \%$ formic acid in water and acetonitrile could improve peak shapes under the optimized conditions. The retention time of IS and poliumoside were $1.91 \mathrm{~min}$ and $1.73 \mathrm{~min}$ for pharmacokinetic study, respectively.

Appropriate extraction method of the plasma is essential for the quantitative analysis. The technique of liquid-liquid extraction and precipitation of protein was tested for alternative. The method of liquid-liquid extrac- 
tion showed low recovery (30\%) when ethyl acetate was used as extraction solvents, and this method was both complicated and time consuming. Various protein-precipitating reagents, such as methanol, acetonitrile and 10\% TCA, were tested. Strong response value, good recovery and clear supernatant for the analytes were observed by using 10\% TCA as the protein-precipitating reagent. Comparing with the neutral or alkaline conditions, poliumoside was found more stable in acidic solvents [11]. Analyte was more stable in 10\% TCA (as acidic solvent) than in the other two precipitant at room temperature, so $10 \%$ TCA was elected to be the most suitable proteinprecipitating reagent in the experiment.

\subsection{Method Validations}

\subsubsection{Selectivity}

Figure 2 presents typical chromatograms of blank plasma, the blank plasma sample spiked with poliumoside and IS, and test plasma sample obtained at 45 min after an intravenous administration of poliumoside. No significant interfering endogenous peaks were observed at the retention time of analyte and IS under the current chromatographic and MS conditions, demonstrating that this method can accurately differentiate and quantify the analyte of the plasma samples.

\subsubsection{Linearity and LLOQ}

The calibration standards of poliumoside $(50,100,625,1250,2500,5000,10,000 \mathrm{ng} / \mathrm{mL})$ were obtained by spiking blank plasma with appropriate amounts of working solutions to the final concentration range of 50 $10,000 \mathrm{ng} / \mathrm{mL}$. Figure 3 showed the calibration curve that was constructed by plotting the peak area ratio of poliumoside to IS versus concentrations of analyte. Linear equation of the calibration curve was: $y=0.0749 x-$ $0.0054\left(r^{2}=0.993\right)$ within the concentration from 50 to $10,000 \mathrm{ng} / \mathrm{mL}$. The LLOQ for the analyte was confirmed at $50 \mathrm{ng} / \mathrm{mL}$ and the limit of detection was found to be $13 \mathrm{ng} / \mathrm{mL}$.

\subsubsection{Precision and Accuracy}

Precision and accuracy at three concentration levels of poliumoside were shown in Table 1 . The intra-day and inter-day precision of QC samples were all less than $7.97 \%$. The accuracy ranged from $-7.01 \%$ to $3.36 \%$ for all the three concentration levels. The results were found to be within the acceptance criteria, demonstrating that the method were reproducible and reliable.

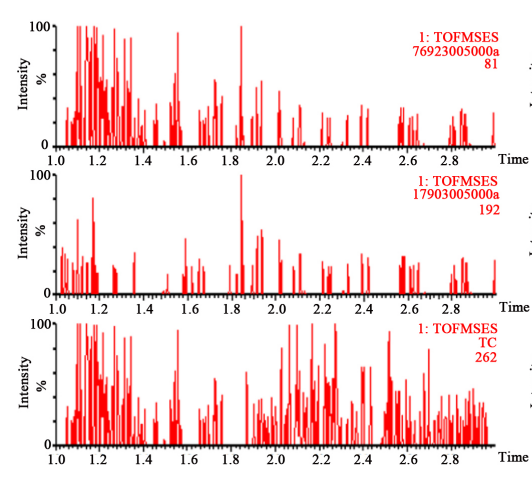

(a)

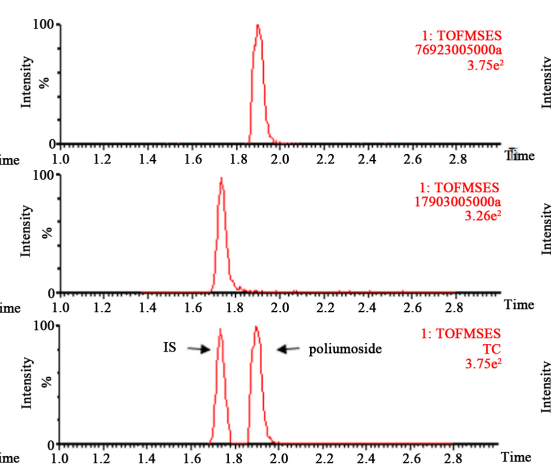

(b)

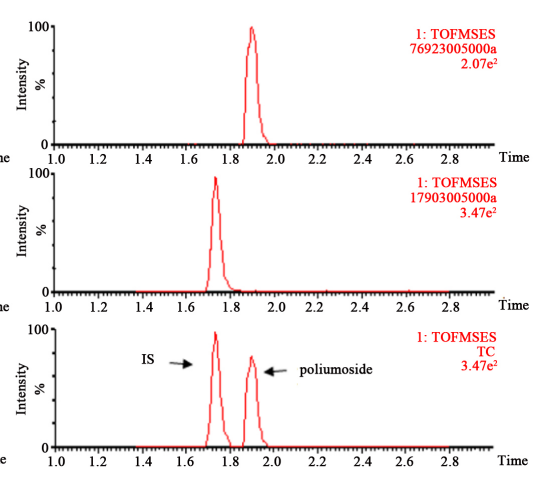

(c)

Figure 2. Representative UPLC-MS chromatograms: (a) Blank plasma; (b) Blank plasma spiked with the poliumoside and IS; (c) A rat plasma sample of $45 \mathrm{~min}$ after intravenous administration of poliumoside $(10 \mathrm{mg} / \mathrm{kg})$.

Table 1. Precision and accuracy for poliumoside in rat plasma $(n=6)$.

\begin{tabular}{ccccccc}
\hline \multirow{2}{*}{$\begin{array}{c}\text { Concentration } \\
(\mu \mathrm{g} / \mathrm{mL})\end{array}$} & Measured conc & RSD (\%) & RE (\%) & Measured conc & RSD (\%) & RE (\%) \\
\cline { 2 - 6 } & $0.196 \pm 0.010$ & 5.10 & -2.00 & $0.186 \pm 0.012$ & 6.45 & -7.00 \\
1 & $1.013 \pm 0.029$ & 2.86 & 1.30 & $0.953 \pm 0.076$ & 7.97 & 3.46 \\
8 & $8.095 \pm 0.288$ & 3.56 & 1.19 & $8.269 \pm 0.286$ & 3.36 \\
\hline
\end{tabular}




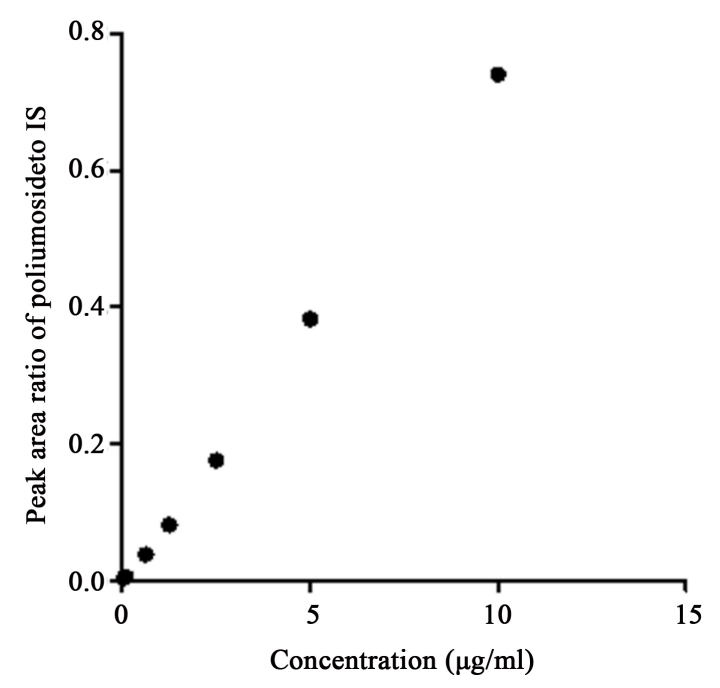

Figure 3. The calibration curve of poliumoside at the concentration range of $50-10,000 \mathrm{ng} / \mathrm{mL}$.

\subsubsection{Recovery and Matrix Effect}

The recovery and matrix effect of poliumoside and IS were shown in Table 2. The mean recovery of three concentrations was between $93.89 \%$ and $103.32 \%$. Matrix effect is the suppression or enhancement of ionization of analytes by the presence of matrix components in the biological samples [17]. The matrix effect ranged from $92.49 \%$ to $105.34 \%$. Partial data of recovery and matrix effects was greater than $100 \%$, and this phenomenon may result from the ion enhancement effect of the matrix for poliumoside. We can conclude that no significant signal suppression or enhancement were observed in the present study. These results were within the acceptable range and indicated that the method were dependable.

\subsubsection{Stability}

Table 3 summarized all established stability for the analyte subjected to different conditions. The RE values were between $-5.0 \%$ and $13.6 \%$ under low temperature conditions. The RE values of the analyte at room temperature were between $-21.07 \%$ and $-44.04 \%$, indicating that poliumoside was unstable under this condition. The results demonstrated that the poliumoside samples kept stable under the low temperature conditions and it was also the guidance for the sample preparation disposing on the ice. Consequently, the developed method was suitable for the pharmacokinetic study of poliumoside.

\subsection{Pharmacokinetic Study}

The plasma concentration-time profiles of poliumoside were shown in Figure 4. The concentration of poliumoside was calculated using the following equation:

$$
\text { Concentration of poliumoside }=\frac{A+0.0054}{0.0749}
$$

$A$ is the peak area ratio of poliumoside to IS from different samples. The mean pharmacokinetic parameters from non-compartment modeling were listed in Table 4. All the pharmacokinetic parameters were calculated by PK solver software. The validated method was successfully applied to measure the plasma concentration of poliumoside in rats. After oral $(200 \mathrm{mg} / \mathrm{kg})$ administration of poliumoside, the $T_{\max }$ was $30 \mathrm{~min}$, suggesting that poliumoside was rapidly absorbed through the gastrointestinal (GI) tract. However, the calculated $C_{\max }$ was only $561 \mathrm{ng} / \mathrm{mL}$ and the low absorbing may result from the high polarity of the structure of poliumoside. Plasma concentration experienced rapidly decline after peak concentration. The fast tissue distribution and the sluggish overall elimination may give rise to the result. The $\mathrm{AUC}_{0-t}$ was $146 \pm 9.88 \mu \mathrm{g} / \mathrm{mL}^{*} \mathrm{~min}$, the mean residence time (MRT) of the analyte after oral and intravenous administration were $15.41 \pm 4.17 \mathrm{~h}$ and $0.74 \pm 0.29 \mathrm{~h}$, respectively. 


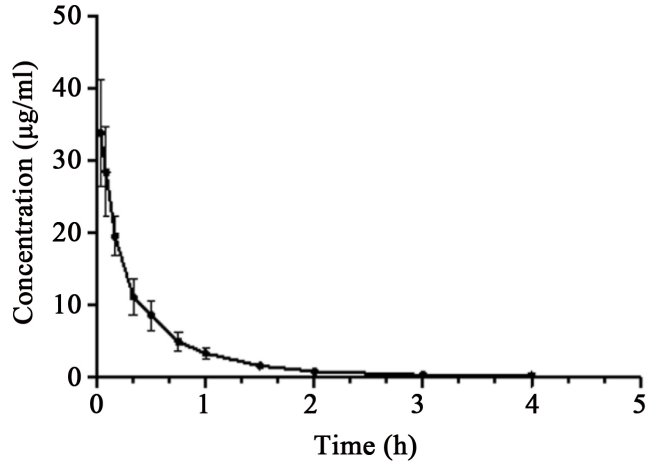

(a)

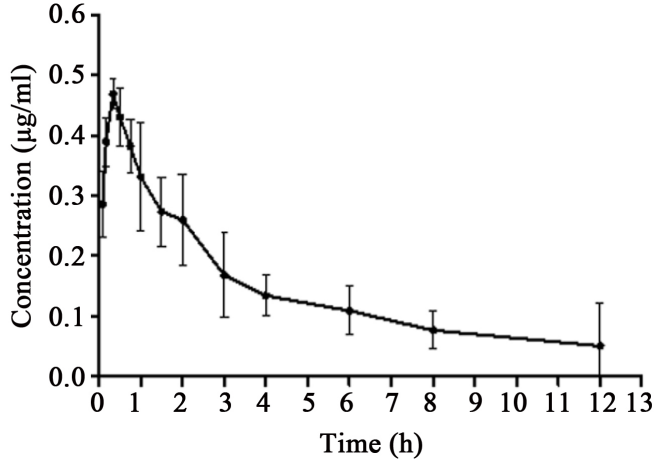

(b)

Figure 4. (a) Mean plasma concentration time profile after intravenous (10 $\mathrm{mg} / \mathrm{kg}$ ) administration of poliumoside in rats; (b) Mean plasma concentration time profile after oral $(200 \mathrm{mg} / \mathrm{kg})$ administration of poliumoside.

Table 2. Recovery, matrix effect for poliumoside and IS of QC samples in rat plasma $(n=6)$.

\begin{tabular}{cccccc}
\hline Compound & Spiked concentration $(\mu \mathrm{g} / \mathrm{mL})$ & Extraction recovery $(\%)$ & RSD $(\%)$ & Matrix effect $(\%)$ & RSD $(\%)$ \\
\hline Poliumoside & 0.2 & 93.89 & 1.20 & 105.34 & 2.71 \\
& 1 & 103.32 & 7.98 & 92.49 & 14.94 \\
& 8 & 99.92 & 6.10 & 100.27 & 12.65 \\
Caffeic acid & 10 & 93.80 & 3.65 & 94.21 & 4.51 \\
\hline
\end{tabular}

Table 3. Stability of poliumoside under different conditions in rat plasma $(\mathrm{n}=3)$.

\begin{tabular}{ccccccccc}
\hline \multirow{2}{*}{ Concentration $(\mu \mathrm{g} / \mathrm{mL})$} & \multicolumn{2}{c}{ Three freeze-thaw cycles } & \multicolumn{2}{c}{ At ice-water bath for $12 \mathrm{~h}$} & \multicolumn{2}{c}{ At room temperature for $12 \mathrm{~h}$} & \multicolumn{2}{c}{ At $4^{\circ} \mathrm{C}$ for 3 days } \\
\cline { 2 - 9 } & RE (\%) & RSD (\%) & RE (\%) & RSD (\%) & RE (\%) & RSD (\%) & RE (\%) & RSD (\%) \\
\hline 0.2 & 13.60 & 0.67 & -5.00 & 5.35 & -44.05 & 21.78 & 5.95 & 3.51 \\
1 & -2.76 & 2.13 & 7.63 & 5.69 & -40.03 & 9.36 & 1.78 & 2.74 \\
8 & -0.21 & 2.85 & 1.53 & 3.80 & -21.07 & 6.52 & -0.19 & 3.05 \\
\hline
\end{tabular}

Table 4. The mean pharmacokinetic parameters from non-compartment.

\begin{tabular}{|c|c|c|c|c|c|}
\hline \multirow{2}{*}{ Parameters } & \multirow{2}{*}{ Unit } & \multicolumn{2}{|c|}{ Intravenous (10 mg/kg) } & \multicolumn{2}{|c|}{ Oral (200 mg/kg) } \\
\hline & & Mean & SD & Mean & SD \\
\hline $\mathrm{AUC}_{0-\mathrm{t}}$ & $\mu \mathrm{g} / \mathrm{mL}^{*} \min$ & 1044.23 & 201.13 & 146 & 9.88 \\
\hline $\mathrm{AUC}_{0-\infty}$ & $\mu \mathrm{g} / \mathrm{mL} * \min$ & 1131.45 & 159.52 & 325.33 & 69.73 \\
\hline MRT & $\mathrm{h}$ & 0.74 & 0.29 & 15.41 & 4.17 \\
\hline$T_{\max }$ & $\min$ & 29.72 & 13.87 & l & l \\
\hline$C_{\max }$ & $\mathrm{ng} / \mathrm{mL}$ & 561.47 & 100.33 & l & l \\
\hline$t_{1 / 2}$ & $\mathrm{~h}$ & 0.85 & 0.19 & 10.47 & 3.25 \\
\hline AUMC $_{0-\infty}$ & $\mathrm{H}^{*} \mathrm{~h} * \mathrm{ng} / \mathrm{mL}$ & 9589.94 & 1369.04 & 98857.22 & 43873.27 \\
\hline V & $\mathrm{L} / \mathrm{kg}$ & 1.91 & 1.78 & 24.18 & 2.94 \\
\hline CL & $\mathrm{L} / \mathrm{h} / \mathrm{kg}$ & 0.92 & 0.3 & 1.68 & 0.41 \\
\hline Bioavailability, F & & & $0.69 \%$ & & \\
\hline
\end{tabular}

Abbreviations: $\mathrm{AUC}_{0-t}$, area under the concentration-time curve calculated from zero up to the last measured concentration; $\mathrm{AUC}_{0-\infty}$, area under the concentration-time curve extrapolated from zero up to infinity; MRT, mean residence time of the analysis; $T_{\max }$, time to maximum observed concentartion; $C_{\max }$, maximum plasma concentration; $t_{1 / 2}$, elimination; $\mathrm{AUMC}_{0-\infty}$, area under the first moment of the concentration-time curve from zero up to infinity; $V$, volume of distribution; CL, clearance. 
The systematic pharmacokinetics of poliumoside in rats was explored for the first time. Furtherly, the bioavailability of poliumoside was firstly reported to be $0.69 \%$, which is similar to the other PhGs [18]-[20]. The metabolism of PhG in GI tract was largely reported, such as poliumoside [21] and echinacoside [22] [23]. The complex microbial ecosystem in GI is generally considered as a separated organ with powerful metabolic capacity. The metabolism in GI may directly decrease absorption of poliumoside and result in the low bioavailability ultimately.

\section{Conclusion}

In conclusion, a rapid, sensitive and selective UPLC/Q-TOF-MS method was established and applied for the determination of poliumoside in rat plasma. Only 3 minutes were needed for the analysis time, and this method truncated the detecting time and improved the accuracy to a large extent. The pharmacokinetic parameters of poliumoside in rats were successfully characterized in this study and the bioavailability was firstly reported to be $0.69 \%$. This study provides a novel approach for pharmacokinetics of poliumoside in rat plasma and provides a basis for further pharmacology research.

\section{References}

[1] Cai, H., Xie, Z., Liu, G., Sun, X., Peng, G., Lin, B. and Liao, Q. (2014) Isolation, Identification and Activities of Natural Antioxidants from Callicarpa kwangtungensis Chun. PLoS One, 9, e93000. http://dx.doi.org/10.1371/journal.pone.0093000

[2] Jia, A., Yang, Y.F., Kong, D.Y. and Xiao, C.C. (2012) GC-MS Analysis of Chemical Constituents of Essential Oil from Callicarpa kwangtungensis and Their Antimicrobial Activity. Zhong Yao Cai, 35, 415-418.

[3] Zhou, G.P., Xie, E.L., Sheng, J.C., Zhang, J., Wang, J. and Yuan, G.P. (2008) HPLC Determination of for Sythoside B and Poliumoside in Callicarpa kwangtungensis Chun. from Different Habitats of Jiangxi. Chinese Journal of Pharmaceutical Analysis, 28, 1623-1625.

[4] Tu, P.F., Shi, H.M., Song, Z.H., Jiang, Y. and Zhao, Y.Y. (2007) Chemical Constituents of Cistanche sinensis. Journal of Asian Natural Products Research, 9, 79-84. http://dx.doi.org/10.1080/10286020500384450

[5] Tai, B.H., Jung, B.Y., Cuong, N.M., Linh, P.T., Tung, N.H., Nhiem, N.X., Huong, T.T., Anh, N.T., Kim, J.A., Kim, S.K. and Kim, Y.H. (2009) Total Peroxynitrite Scavenging Capacity of Phenylethanoid and Flavonoid Glycosides from the Flowers of Buddleja officinalis. Biological and Pharmaceutical Bulletin, 32, 1952-1956. http://dx.doi.org/10.1248/bpb.32.1952

[6] Yu, S.Y., Lee, I.S., Jung, S.H., Lee, Y.M., Lee, Y.R., Kim, J.H., Sun, H. and Kim, J.S. (2013) Caffeoylated Phenylpropanoid Glycosides from Brandisia hancei Inhibit Advanced Glycation End Product Formation and Aldose Reductase in Vitro and Vessel Dilation in Larval Zebrafish in Vivo. Planta Medica, 79, 1705-1709. http://dx.doi.org/10.1055/s-0033-1351101

[7] De Marino, S., Festa, C., Zollo, F., Incollingo, F., Raimo, G., Evangelista, G. and Iorizzi, M. (2012) Antioxidant Activity of Phenolic and Phenylethanoid Glycosides from Teucrium polium L. Food Chemistry, 133, 21-28. http://dx.doi.org/10.1016/j.foodchem.2011.12.054

[8] He, Z.D., Lau, K.M., Xu, H.X., Li, P.C. and Pui-Hay But, P. (2000) Antioxidant Activity of Phenylethanoid Glycosides from Brandisia hancei. Journal of Ethnopharmacology, 71, 483-486. http://dx.doi.org/10.1016/S0378-8741(00)00189-6

[9] Koo, K.A., Sung, S.H., Park, J.H., Kim, S.H., Lee, K.Y. and Kim, Y.C. (2005) In Vitro Neuroprotective Activities of Phenylethanoid Glycosides from Callicarpa dichotoma. Planta Medica, 71, 778-780. http://dx.doi.org/10.1055/s-2005-871213

[10] Tatli, II, Takamatsu, S., Khan, I. and Akdemir, Z.S. (2007) Screening for Free Radical Scavenging and Cell Aggregation Inhibitory Activities by Secondary Metabolites from Turkish Verbascum Species. Zeitschrift fur Naturforschung Section C, 62, 673-678. http://dx.doi.org/10.1515/znc-2007-9-1008

[11] Sun, X., Liao, Q., Liu, G., Cai, H., Zhang, L., Zhu, C. and Xie, Z. (2013) Simultaneous Determination of Three Phenylethanoid Glycosides from Callicarpae Caulis et Folium in Rat Plasma by LC-MS/MS and Its Application to PK Study. Bioanalysis, 5, 1883-1895. http://dx.doi.org/10.4155/bio.13.124

[12] Liu, M.Z., Zhang, Y.L., Zeng, M.Z., He, F.Z., Luo, Z.Y., Luo, J.Q., Wen, J.G., Chen, X.P., Zhou, H.H. and Zhang, W. (2015) Pharmacogenomics and Herb-Drug Interactions: Merge of Future and Tradition. Evidence-Based Complementary and Alternative Medicine, 2015, Article ID: 321091. http://dx.doi.org/10.1155/2015/321091

[13] Cote, C., Bergeron, A., Mess, J.N., Furtado, M. and Garofolo, F. (2009) Matrix Effect Elimination during LC-MS/MS Bioanalytical Method Development. Bioanalysis, 1, 1243-1257. http://dx.doi.org/10.4155/bio.09.117 
[14] Wen, C., Lin, C., Cai, X., Ma, J. and Wang, X. (2014) Determination of Sec-O-Glucosylhamaudol in Rat Plasma by Gradient Elution Liquid Chromatography-Mass Spectrometry. Journal of Chromatography B, 944, 35-38. http://dx.doi.org/10.1016/j.jchromb.2013.11.001

[15] Wang, X., Chen, M., Wen, C., Zhang, Q. and Ma, J. (2013) Determination of Chidamide in Rat Plasma by LC-MS and Its Application to Pharmacokinetics Study. Biomedical Chromatography, 27, 1801-1806. http://dx.doi.org/10.1002/bmc.3001

[16] Zhang, Q., Wen, C., Xiang, Z., Ma, J. and Wang, X. (2014) Determination of CUDC-101 in Rat Plasma by Liquid Chromatography Mass Spectrometry and Its Application to a Pharmacokinetic Study. Journal of Pharmaceutical and Biomedical Analysis, 90, 134-138. http://dx.doi.org/10.1016/j.jpba.2013.11.031

[17] Viswanathan, C.T., Bansal, S., Booth, B., DeStefano, A.J., Rose, M.J., Sailstad, J., Shah, V.P., Skelly, J.P., Swann, P.G. and Weiner, R. (2007) Quantitative Bioanalytical Methods Validation and Implementation: Best Practices for Chromatographic and Ligand Binding Assays. Pharmaceutical Research, 24, 1962-1973. http://dx.doi.org/10.1007/s11095-007-9291-7

[18] Jia, C., Shi, H., Wu, X., Li, Y., Chen, J. and Tu, P. (2006) Determination of Echinacoside in Rat Serum by Reversed-Phase High-Performance Liquid Chromatography with Ultraviolet Detection and Its Application to Pharmacokinetics and Bioavailability. Journal of Chromatography B, 844, 308-313. http://dx.doi.org/10.1016/j.jchromb.2006.07.040

[19] Wu, Y.T., Lin, L.C., Sung, J.S. and Tsai, T.H. (2006) Determination of Acteoside in Cistanche deserticola and Boschniakia rossica and Its Pharmacokinetics in Freely-Moving Rats Using LC-MS/MS. Journal of Chromatography B, 844, 89-95. http://dx.doi.org/10.1016/j.jchromb.2006.07.011

[20] Zhou, W., Tan, X., Shan, J., Liu, T., Cai, B. and Di, L. (2014) Effect of Chito-Oligosaccharide on the Intestinal Absorptions of Phenylethanoid Glycosides in Fructus Forsythiae Extract. Phytomedicine, 21, 1549-1558. http://dx.doi.org/10.1016/j.phymed.2014.06.016

[21] Deng, R., Xu, Y., Feng, F. and Liu, W. (2014) Identification of Poliumoside Metabolites in Rat Feces by High Performance Liquid Chromatography Coupled with Quadrupole Time-of-Flight Tandem Mass Spectrometry. Journal of Chromatography B, 969, 285-296. http://dx.doi.org/10.1016/j.jchromb.2014.08.032

[22] Lei, L., Song, Z.H., Tu, P.F., Li, Y.Z., Wu, L.J. and Chen, F.K. (2001) Metabolic Regulation of Phenylethanoid Glycosides from Herba cistanches in Dogs' Gastrointestine. Acta Pharmaceutica Sinica, 36, 432-435.

[23] Wang, Y., Hao, H., Wang, G., Tu, P., Jiang, Y., Liang, Y., Dai, L., Yang, H., Lai, L., Zheng, C., Wang, Q., Cui, N. and Liu, Y. (2009) An Approach to Identifying Sequential Metabolites of a Typical Phenylethanoid Glycoside, Echinacoside, Based on Liquid Chromatography-Ion Trap-Time of Flight Mass Spectrometry Analysis. Talanta, 80, 572-580. http://dx.doi.org/10.1016/j.talanta.2009.07.027 